\title{
FLORES, ACAPARAMIENTO DEL AGUA Y RESPONSABILIDAD EMPRESARIAL SOCIAL: CERTIFICACIÓN DE LA PRODUCCIÓN DE ROSAS Y RECLAMOS POR LA JUSTICIA AMBIENTAL EN EL ECUADOR
}

\begin{abstract}
Resumen
La producción comercial de flores, el acaparamiento del agua y las luchas sociales por un reparto equitativo y un ambiente sano tienen una estrecha relación. Ecuador es el tercer exportador mundial de rosas. Cayambe-Tabacundo es la principal zona de producción y presenta una fuerte presión sobre los recursos hídricos. El agua es acaparada por las grandes empresas florícolas, quedando el resto para agricultura/ganadería de subsistencia en comunidades indígenas. Las florícolas utilizan las certificaciones ambientales y de comercio justo privadas, pero los estándares son permisivos. Este artículo analiza las relaciones de poder detrás de las prácticas de certificación de flores. Concluye que solo tras la presión de agencias gubernamentales, ONGs y asociaciones de usuarios, las empresas florícolas comenzaron a cumplir los estándares.

PALABRAS CLAVE: certificaciones; responsabilidad social empresarial; justicia ambiental; floricultura; Ecuador

a Departamento de Ciencias Ambientales, Water Resources Management Group, Universidad de Wageningen, P.O. Box 47, 6700 AA Wageningen, Países Bajos. pamv59@gmail.com

b Departamento de Ciencias Ambientales, Water Resources Management Group, Universidad de Wageningen, P.O. Box 47, 6700 AA Wageningen, Países Bajos. jeroen.vos@wur.nl

c Departamento de Ciencias Ambientales, Water Resources Management Group, Universidad de Wageningen, P.O. Box 47, 6700 AA Wageningen, Países Bajos. pippi.vanommen@wur.nl

d Department of Environmental Sciences, Wageningen University, The Netherlands. rutgerd.boelens@wur.nl CEDLA Centre for Latin American Research and Documentation, The Netherlands.

Department of Geography, Planning and International Development Studies, University of Amsterdam, The Netherlands.
\end{abstract}

Fecha de recepción: octubre 2018. Fecha de aceptación: diciembre 2018. 


\section{Abstract}

\section{FLOWERS, WATER ACCUMULATION AND CORPORATE SOCIAL RESPONSIBILITY: CERTIFICATION OF THE PRODUCTION OF ROSES AND CLAIMS FOR ENVIRONMENTAL JUSTICE IN ECUADOR}

Commercial production of flowers, accumulation of water, and social struggles for equitable distribution and a healthier environment have an intimate relationship. Ecuador is the third world exporter of roses. Cayambe-Tabacundo is the main production region and counts with major water conflicts. Large floriculturists accumulate water, leaving the rest for subsistence agriculture and livestock in indigenous communities. Floriculture uses private environmental and fair-trade certification, but the standards are permissive. This article analyzes the power relations that steer flower certification practices. It concludes that only after pressure from government agencies, NGOs and user associations, floriculture began to meet the standards.

KEYwORDS: certifications; corporate social responsibility; environmental justice; floriculture; Ecuador

\section{INTRODUCCIÓN}

En la mayoría de las regiones del mundo se está viviendo un gran auge en el comercio internacional de productos agrícolas. Políticas gubernamentales para la expansión de cultivos intensivos en agua a menudo han conllevado una acumulación de derechos de agua por parte de grandes empresas agrícolas. Estos procesos de expansión tienen en común que compiten por agua y tierra con pequeños productores locales e indígenas, degradan los ecosistemas locales y la salud humana y ambiental, vulneran la seguridad alimentaria local, y alteran profundamente los modos existentes de producción y la distribución de ingresos (Lynch, 2012; Vos y Boelens, 2014; Mena et al., 2016; Hidalgo et al., 2017). Para las comunidades campesinas y los pueblos indígenas esta degradación ambiental y despojo legal, extralegal o ilegal — no solo de sus tierras sino sobre todo de sus fuentes de agua- suele hacer imposible seguir viviendo en su hábitat acostumbrado (Hoogesteger, 2012; Roa-García, 2014).

En este artículo analizamos el despojo del agua por la intrusión del sector de la exportación florícola que amenaza las comunidades indígenas en la sierra ecuatoriana y, en particular, la certificación para productos agrícolas de exportación por parte de empresas certificadoras privadas (como para las flores de exportación) como elemento clave de la Responsabilidad Social Empresarial (RSE) de las cadenas de suministro mundiales para monitorear y mejorar sus prácticas socioambientales. Las empresas tienen tres razones para certificar. Primero, los productores deben garantizar la calidad, y los estándares deben prevenir el agotamiento y la degradación de los recursos que podrían poner en peligro la producción. Segundo, la reputación de los supermercados en el Norte global — donde finalmente se vende la producciónes vulnerable, y productos de baja calidad, riesgos para la salud y desastres ambientales po- 
drían dañar su imagen. Tercero, para las cadenas el comercio justo y los productos «verdes» son un mercado creciente en muchos países (Hughes, 2001).

Para organizar el sistema de monitoreo, la certificación privada tiene generalmente los siguientes elementos: un documento público que detalla las normas; una auditoría realizada por terceros privados; un sello en el producto para notificar al consumidor o una certificación utilizada por los minoristas, y una organización de certificación que establece y comunica los estándares, capacita a productores e inspectores, y autoriza a los agentes de auditoría (Mutersbaugh et al., 2005). Aun así, no obstante los discursos empresariales y su elogio a los esquemas de certificación, investigadores han levantado dudas sobre la efectividad de la certificación privada en la práctica de las empresas (Langen y Adenaeuer, 2013; Raynolds, 2014). Vos y Boelens (2014) presentan seis críticas sobre la certificación privada: a) la definición tecnocrática y uniforme de estándares (no adaptada al contexto local); b) los bajos niveles de transparencia y democracia; c) la exclusión de pequeños propietarios (tarifas altas, estándares no adaptables); d) los beneficios ambientales insignificantes (los «buenos» ya realizan buenas prácticas, y los «malos» no las adoptan); e) el riesgo de cooptación y criterios laxos y f) las dificultades en la auditoría, en particular, la susceptibilidad ante el fraude y auditorías deficientes.

El artículo examina la contribución de las certificaciones al cambio socioambiental en una cadena de suministro para el caso de la floricultura en Ecuador. Desde que estas actividades comenzaron en los Andes norteños a principios de los 1980s, el Ecuador se ha convertido en el tercer exportador mundial de flores (UN Comtrade, 2018). La producción, principalmente rosas, tiene un enorme impacto en la economía de la región Cayambe-Tabacundo al norte de Quito, que corresponde a la cuenca del río Pisque (Figura 1). El sector emplea mucha gente de las comunidades indígenas, proporcionando un medio de vida, pero infligiendo cambios culturales en términos, por ejemplo, alimentarios y de relaciones intrafamiliares. Se han planteado preocupaciones con respecto a las condiciones de trabajo, incluidos efectos en la salud por los plaguicidas, y efectos ambientales generales (Tenenbaum, 2002; Sawers, 2005; Breilh et al., 2007, 2009). Aunque muchos entrevistados declararon que el uso de pesticidas ha menguado (véase también Soper, 2013), Victor van Dijk, Gerente para Sudamérica de FleuraMetz, declaró: «Nos esforzamos por obtener 90 \% de flores producidas sosteniblemente. Pero en Ecuador todavía queda un largo camino» (Bloemisterij, 2017, traducción propia).

En el caso estudiado en la región de Cayambe-Tabacundo, la creciente escasez de agua de riego generó un conflicto sobre el uso de un canal compartido entre pequeños y grandes productores. Los pequeños propietarios obtuvieron el control del sistema de riego en 2006 y lograron acceso a más agua, pero la mayor parte aún se destina a grandes compañías. Ahora, los 2.500 pequeños usuarios usan un total de $2 \mathrm{hm}^{3} /$ año, mientras los 100 florícolas grandes utilizan un total de $8 \mathrm{hm}^{3} /$ año (Mena-Vásconez et al., 2017). Paradójicamente, desde 2005 muchos pequeños productores han establecido sus propios microinvernaderos y venden flo- 
res a comerciantes o grandes productores de flores. Más de 600 pequeños productores venden flores para exportar a comerciantes internacionales, con sus pequeñas fincas dispersas entre las más grandes (Figura 2) (Mena-Vásconez et al., 2016). Esta serie de cambios en la producción, la sociedad y el ambiente en la región ha llevado a la pregunta central de este texto: ¿Cual es la influencia relativa de la certificación privada en las transformaciones socioambientales en esta región florícola del Ecuador?

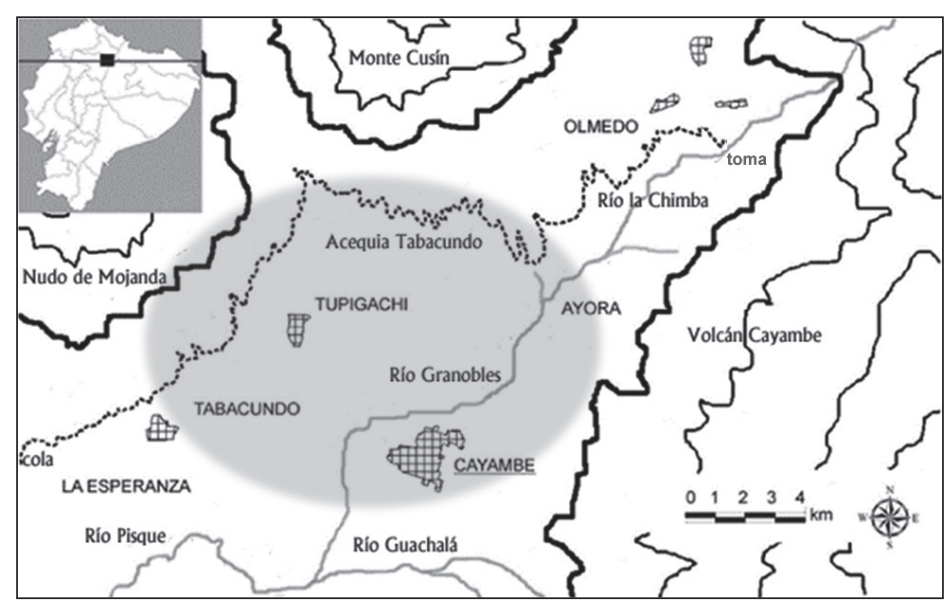

Figura 1. Visión general del área de estudio, con su localización en el Ecuador. El óvalo sombreado indica la zona de concentración de las florícolas (modificado de Mena-Vásconez et al., 2016).

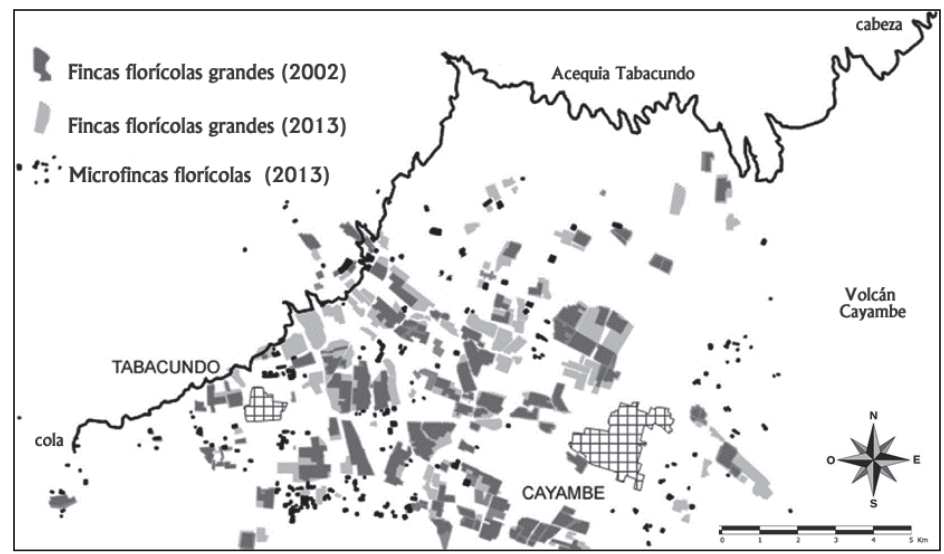

Figura 2. Distribución de las fincas grandes en 2002 y 2013, y las microflorícolas en 2013. En 2002 no había microflorícolas; el área corresponde aproximadamente al óvalo señalado en la Figura 1 (adaptado de Mena Vásconez et al., 2016). 


\section{LAS DIMENSIONES DE PODER QUE CARACTERIZAN LA CERTIFICACIÓN:}

\section{NOCIONES CONCEPTUALES}

En este artículo estudiamos los intereses específicos de las partes mediante un análisis de poder enfocado en la creación y aplicación de regulaciones en la floricultura ecuatoriana. Para ello, entendemos las normas socioambientales no como reglas objetivas aplicadas por organizaciones para el beneficio general, sino como insumos y resultados que se forman y que se aplican en una red de poderes. Estas normas regulan los actores, sus visiones y sus prácticas. Por lo tanto, observamos los discursos dominantes que legitiman la estructura de representación y las normas y valores aceptados en la gobernanza de la floricultura. Coincidimos con Lemke (2007: 58, traducción propia) en que, para comprender el cambio social, debemos estudiar «prácticas en lugar de objetos, estrategias en lugar de funciones y tecnologías en lugar de instituciones». Así, estructuraremos este artículo según estrategias, prácticas y tecnologías de cinco agentes clave en el negocio: a) certificadores y auditores, b) grandes productores, c) pequeños productores, d) organizaciones gubernamentales y e) ONG.

Un marco conceptual para investigar las posibilidades de cambio social es el análisis de las formas y dimensiones de poder, según las ideas de Gaventa (2006) y su «cubo de poder» con base en teorías weberianas/(neo)marxistas y foucaultianas. El análisis tridimensional del cubo presenta espacios de participación (arenas políticas), niveles de interacción (escalas socio-geográficas) y formas de poder (modos y expresiones de poder) (Figura 3).

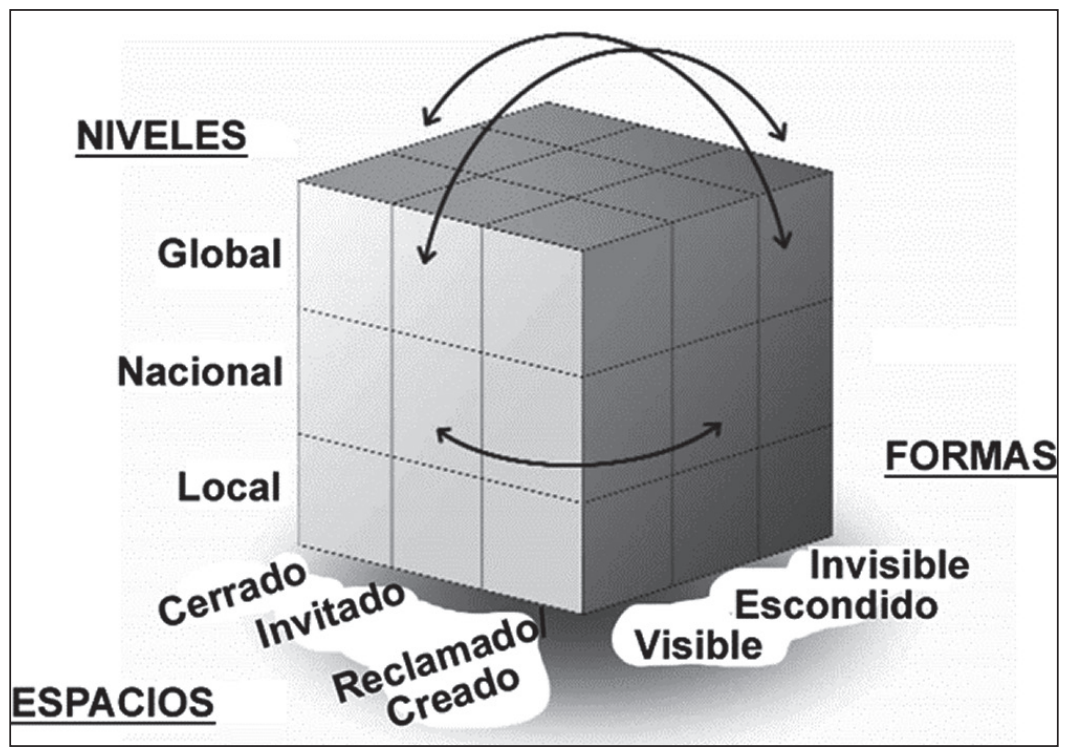

Figura 3. El «cubo de poder» (modificado de Gaventa, 2006). 
Hay tres espacios de poder: cerrados, invitados y reclamados. Los cerrados son procesos de decisión donde los responsables — por ejemplo, burócratas o expertos- deciden sin que participen los actores interesados/afectados. En los espacios invitados, los responsables invitan a los interesados/afectados a participar en la decisión. Su grado de opinión varía desde ser informados hasta tener una voz «asignada»; los temas y las reglas son establecidos por los organizadores. Los espacios reclamados (exigidos) existen cuando los grupos de la sociedad civil influyen realmente y pueden determinar la agenda y las reglas.

Los niveles de poder se refieren a las escalas de gobernanza, desde lo global a lo local. Las instituciones y reglamentaciones se definen cada vez más globalmente. Las organizaciones locales aplican estrategias enraizadas y de abajo hacia arriba para defenderse contra reglas universalizantes que vienen desde arriba.

Las tres formas de poder se refieren al tipo de poder en una escala y espacio dados. El poder visible son estructuras políticas oficiales, reglas formales y procedimientos de decisión. El poder oculto es una forma que, en principio, es visible, pero es ocultado activamente por personas y organizaciones poderosas. Este poder funciona al excluir a otros del establecimiento de la agenda para discutir y tomar decisiones. El poder invisible funciona a través de la formación de significados y verdades, y lo que es aceptable, deseable y normal. Esto es lo que Foucault (1995) llamó modo de poder normalizador o disciplinario (y resulta en formas de «gubernamentalidad», Foucault, 1991; Baumgarten y Ullrich, 2012; Boelens et al., 2015): este poder invita (a menudo inconscientemente o sin intencionalidad expresa) a internalizar lo deseado, normal, porque no hacerlo evocaría inmoralidad y sentimientos de culpabilidad. Examinar las prácticas de certificación de flores a través de estas dimensiones ayudará a examinar como, donde y porque se diseñan y aplican las regulaciones socioambientales.

\section{MÉTODOS}

Los datos se recopilaron entre 2012 y 2018, con más intensidad entre octubre de 2012 y octubre de 2014. Se aplicó un cuestionario a 53 representantes de diversas florícolas de la región. Se realizaron entrevistas semiestructuradas a 23 representantes de pequeñas y medianas florícolas. Durante todo el período se llevaron a cabo aproximadamente 40 entrevistas abiertas con propietarios de pequeñas y grandes empresas y representantes de los gobiernos locales de los cantones Pedro Moncayo y Cayambe en la provincia de Pichincha, ONG como CESA, asociaciones de usuarios, comerciantes, asociaciones de productores, certificadores y cultivadores (breeders). Esto se complementó con una investigación bibliográfica, de archivos y medios de prensa. La información recabada incluía preguntas relacionadas con las certificaciones en el sentido de conocimiento general sobre el tema, pertenencia a algún esquema, experiencias, opiniones y expectativas. 


\section{LOS RETOS DE LA PRODUCCIÓN FLORÍ́COLA EN ECUADOR}

En Ecuador, como en los países vecinos, el limitado acceso al agua no es consecuencia únicamente de factores naturales y climáticos, sino principalmente de los concernientes con relaciones de poder económico y político que entran en juego entre los usuarios que compiten por el recurso en un territorio hídrico determinado (Boelens et al., 2014, 2015; Hoogesteger, 2012; Hidalgo et al., 2017; Rodríguez-de-Francisco y Boelens, 2016). Una dimensión reciente e importante de esta competencia es la intrusión del sector florícola, con impactos no solo en la gobernanza local del agua sino en muchos ámbitos. La producción de flores ecuatorianas comenzó a principios de los 1980s en manos mayormente extranjeras, aprovechando un clima favorable, numerosas horas de sol, noches frías y gran altitud, mano de obra barata, regulación ambiental permisiva, baja imposición fiscal y proximidad al aeropuerto (Soper, 2013; Mena-Vásconez et al., 2017). La calidad y el tamaño de las rosas ecuatorianas son mundialmente famosos. Las exportaciones han aumentado de 195 millones de dólares en el año 2000 a 881 millones en el año 2017 (UN Comtrade, 2018).

La mayoría se produce en Cayambe y Tabacundo, a una hora al norte de Quito y a una altitud de $2.800 \mathrm{~m}$, donde algunas haciendas han permanecido desde la época colonial junto a comunidades. El sector emplea a más de 100.000 trabajadores (USDA, 2009), la gran mayoría indígena, 60 \% mujeres (Korovkin, 2003). El agua de riego es escasa; la Acequia Tabacundo, principal sistema de riego (véanse Figuras 1 y 2), ha sido eje de duras batallas. En 2006 el sistema fue asumido por la asociación de usuarios de agua CODEMIA, que es controlada por comunidades campesinas (Mena-Vásconez et al., 2017).

La industria florícola ecuatoriana se puede dividir en «grandes» y «pequeñas» empresas. Hay alrededor de 300 grandes ( $>2$ ha) y cerca de $700(<2$ ha) empresas familiares de flores (Mena-Vásconez et al., 2017). La mayoría de las grandes están en antiguas haciendas y actualmente son principalmente ecuatorianas, pero también hay holandesas, alemanas, estadounidenses y colombianas. La exportación se realiza principalmente a EEUU, Rusia y Europa. El tradicional paisaje agrícola se ha ido transformando y ahora presenta una mezcla de zonas urbanas, plantaciones de eucalipto, remanentes de zonas de cultivo y un creciente mar de invernaderos de flores cubiertos de plástico blanco (Figura 4a). 

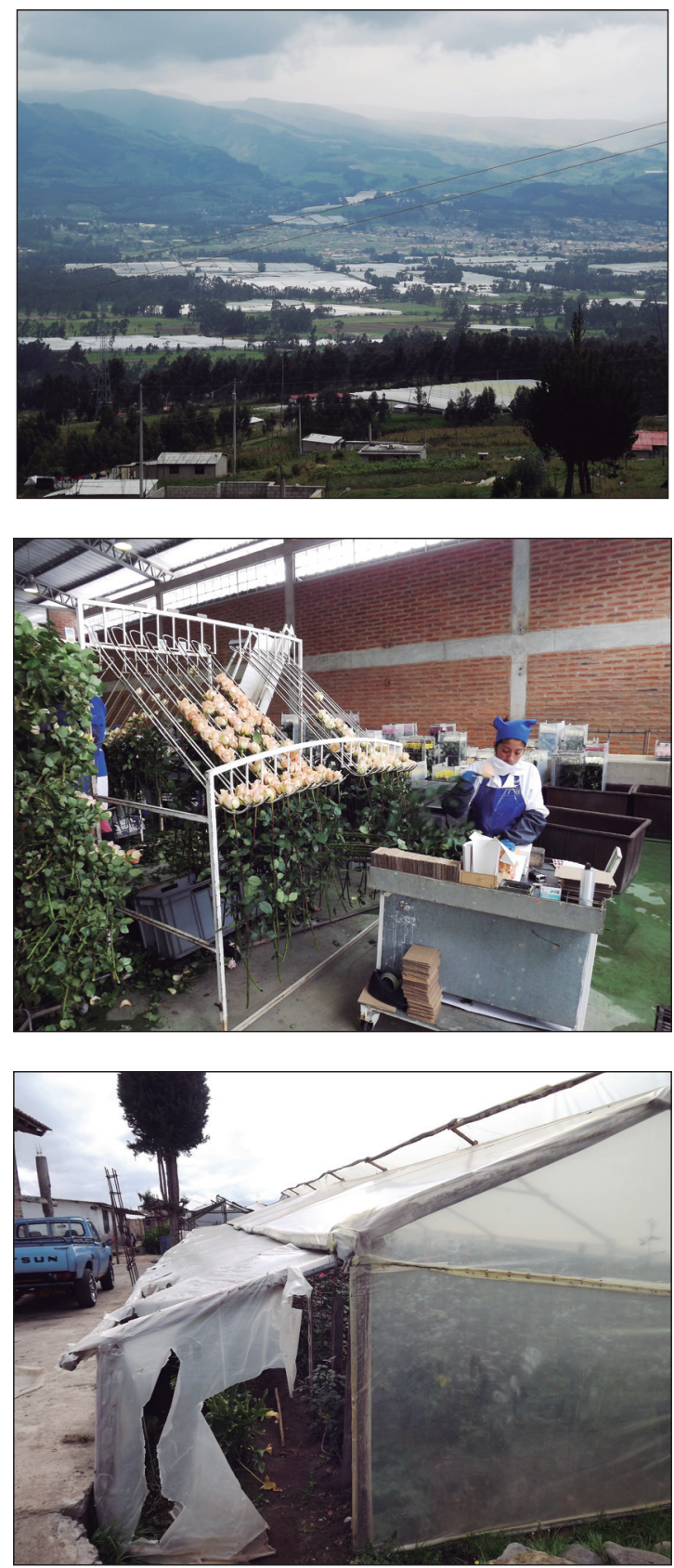

Figura 4. a) Visión parcial de la cuenca del Pisque con dominancia de florícolas grandes b) facilidades de poscosecha en una florícola grande c) Una microflorícola (Fotografía: Patricio Mena Vásconez, 2014) 
Los invernaderos son armazones de acero o eucalipto cubiertos con plástico. El riego es por goteo; cuanto más pequeño el negocio, más simple el sistema de riego. Los agroquímicos usados se disuelven en el agua. Las granjas grandes incluyen instalaciones de poscosecha (acopio, procesamiento, cámaras frigoríficas), mientras que la mayoría de las pequeñas venden a acopiadores (Figura 4b). Del mismo modo, las instalaciones grandes poseen transporte especializado, mientras que las pequeñas deben contratarlo. Desde 2005, cientos de campesinos han establecido florícolas gracias a créditos, experiencia y conocimiento adquiridos en las empresas (Mena-Vásconez et al., 2017). Son de gestión familiar, pero pueden emplear uno o más trabajadores. La compañía capitalista, a su vez, compra y exporta las flores marcándolas como propias, reduciendo así los costos y los riesgos. Los incipientes intentos de asociación han sido mayormente infructuosos (ver abajo).

La floricultura ecuatoriana ha generado impactos severos por el uso de pesticidas (Korovkin, 2003; Breilh, 2007; Soper, 2013). Las flores se han cultivado con productos prohibidos en Europa y Norteamérica (como Carbofurán, Diazimon y bromuro de metilo) que causan enfermedades y alteraciones, algunas graves como aumentos en la tasa de infertilidad, abortos y deformaciones congénitas debido al uso de órgano-fosfatos y otros pesticidas tóxicos (Korovkin, 2003, 2005; Breilh, 2007; Franze y Ciroth, 2011). Por esto y la denegación de servicios de salud, salarios inferiores al mínimo y horas extras obligatorias, la industria ha sido descrita como dura y explotadora (Tenenbaum, 2002; Sawers, 2005). No solo los trabajadores han sido afectados, sino también la comunidad, así como el suelo y el agua (IEDECA, 1999; Breilh, 2007; Breilh et al., 2009).

La floricultura requiere mucha agua para el riego. Esto ha hecho que surjan conflictos en comunidades con florícolas sobre el acceso al recurso (Mena-Vásconez et al., 2016, 2017; Hidalgo et al., 2017). También se ha dicho que la floricultura tiene un impacto negativo en las relaciones sociales en las comunidades donde ha habido «erosión de la organización comunal» (Korovkin, 2003) por procesos individualistas de producción y un agronegocio orientado a beneficios, chocando con las normas comunitarias (Mena-Vásconez et al., 2016; Hidalgo et al., 2017). Una publicación del grupo indígena Cayambi ${ }^{1}$ (GADIP, 2015, 71) afirma:

«No solo la floricultura en sí misma ha desgarrado a los Cayambis, sino que la falta de un mercado justo ha frenado las empresas comunitarias de la población. La hegemonía de un comercio injusto favorece a los grandes productores...».

En cierta manera, las condiciones de trabajo y el impacto ambiental de la producción de flores han mejorado (Soper, 2013) por el surgimiento de un mercado consciente en Oc-

1 Los cayambis o kayambis son un grupo étnico que, junto a Caranquis, Otavalos y Cochisquíes, constituyeron una confederación en el norte de Quito que, tras enfrentarse por 15 años a los Incas, fue vencida en la cruenta batalla de Yahuarcocha en 1487. En la actualidad forman parte del pueblo quichua de la Sierra (Ayala Mora, 2008). 
cidente que ha estimulado el crecimiento de organizaciones como Fairtrade International y Rain Forest Alliance. Estas comenzaron a trabajar con florícolas, asegurando al consumidor que las condiciones de trabajo cumplieran con los estándares. Otros factores han contribuido también a mejorar el proceso de producción de flores: (1) aumento del salario mínimo oficial; (2) lucha de las comunidades campesinas y asociaciones de usuarios por la reasignación de agua; (3) presión de ONG locales; y (4) regulaciones ambientales a varias escalas. De manera general, recientemente el compromiso en el sector para combatir los problemas descritos ha aumentado. Por otro lado, el gobierno ha promulgado regulaciones. Organizaciones internacionales han creado normas privadas para reducir el impacto socioambiental de la floricultura. Algunas compañías también desean entrar en el mercado «verde». Veamos una descripción de los diferentes estándares para la certificación en floricultura, y cómo se hace el proceso de auditoría de la certificación.

\section{LA CERTIFICACIÓN DE FLORES}

En términos generales, los estándares se establecen de manera vertical a una escala global en un «espacio cerrado» según el «cubo de poder» presentado arriba. Estas prácticas normalizan la idea de regulación a través de la competencia en el mercado global. Esto empodera a las compañías que establecen los estándares a través del «poder invisible» del discurso neoliberal. Las compañías ganan poder al obtener acceso al mercado el nicho de productos certificados (con monitoreo permisivo), legitiman el uso y la concentración del agua (porque son «eficientes») y establecen normas sobre la producción (Boelens y Vos, 2012). Así, la certificación fortalece la universalización de las normas de «eficiencia» del uso del agua. Esta es una forma de poder oculto: los organismos de certificación predeterminan la agenda para la discusión sobre los impactos de la floricultura. Se convierte en una forma de poder «invisible»: las normas son aceptadas e internalizadas por los agricultores pequeños que comienzan a considerarse «ineficientes» (y así, moralmente culpables) y aceptan el uso del agua acumulada por las grandes florícolas (Vos y Boelens, 2014, 2018).

A continuación describimos cinco estándares privados frecuentemente utilizados. La mayoría de las grandes compañías entrevistadas exportan flores con una o más certificaciones, aunque algunas informaron que no se involucran o no necesitan certificación. El Flower Label Program (FLP) fue el único programa que desarrolló estándares con la participación de sindicatos, organizaciones ambientales, agricultores, gobiernos y organizaciones de consumidores. En otros casos, las normas fueron determinadas por la empresa normadora, con alguna consulta a los interesados. La certificación es relevante para empresas que exportan a países del norte de Europa y EE.UU. Otros países, como Rusia, China, pero también España, no requieren certificación. Un dueño de una florícola dijo: 
«En Rusia y China no se preocupan por condiciones de trabajo, no necesitan certificaciones para probar nada. En Estados Unidos les importa un poquito, pero es más en Europa donde están conscientes y exigen sostenibilidad» (comunicación personal, 11 de mayo de 2017).

Un exagente comercial responde a la pregunta sobre la sostenibilidad y la demanda del mercado: «No, no, no hacemos absolutamente nada con la sostenibilidad. Realmente no importa en nuestro negocio, no es un tema. [...]» (Marcelo Vallejo, comunicación personal, 13 de mayo de 2017). Las florícolas pueden decidir a que mercado enfocarse. Muchas venden a Rusia, un país desinteresado en normas socioambientales. Sin embargo, algunos productores han extendido su riesgo vendiendo a EE.UU., donde las flores certificadas son un mercado creciente. Aproximadamente un tercio de los comerciantes de flores en EE.UU. requiere certificación. Un ex inspector del FLP notó que muchos gerentes de las grandes florícolas obtuvieron una certificación por la presión de sus pares: «si el vecino tiene una certificación, yo también quiero» (Orlando Felicita, comunicación personal, 2 de abril de 2018). Seguidamente detallamos los esquemas de certificación más aplicados en las florícolas en Ecuador:

\section{Flower Label Program}

Creado en 1999 en Alemania, esta iniciativa de certificación de mesa redonda creada por Brot für die Welt, FIAN y Terre des Hommes funcionó en varios países exportadores de flores como Ecuador, Colombia y Kenia. En Ecuador trabajaron con la ONG CEAS. En 2006 había 56 florícolas ecuatorianas certificadas con FLP (SIPAE, 2016). Se detuvo en 2016 porque el esquema de certificación no funcionó con auditoría de terceros. CEAS tuvo éxito al poner en la agenda los efectos sobre la salud del uso de pesticidas en la floricultura. Presionaron a las florícolas para reducir su uso e introducir medidas para proteger a los trabajadores de la exposición a pesticidas en invernaderos. También presionaron al gobierno para que regulara su uso (Breilh, 2007; Breilh et al., 2009).

\section{FlorEcuador}

Es una certificación propia de Expoflores, una asociación de grandes compañías fundada en 2007. Dicen cubrir el $80 \%$ de las flores exportadas. Todas sus empresas tienen más de 15 ha. Expoflores solo reconoce la existencia de alrededor de cien compañías de flores, de las cuales representan aproximadamente las treinta mayores. Los miembros pagan una tarifa mensual dependiendo del área de invernaderos. Cada tres meses se reúnen en una asamblea. La asociación no está a cargo de la exportación; los miembros tienen sus clientes e instalaciones de poscosecha. Según el representante local en Cayambe, «la ventaja de trabajar como asociación es que puedes hacer más cosas, compartir información y hacer mejores negocios con, por ejemplo, el gobierno» (comunicación personal, 10 de mayo de 2017). El estándar FlorEcuador nació en 2007. Actualmente, para ser parte de Expoflores se requiere esta certi- 
ficación. FlorEcuador tiene nueve criterios obligatorios y 121 criterios, de los cuales se debe cumplir 80 \% (Expoflores, 2016a). Entre los obligatorios destacan dos: la prohibición del trabajo infantil por debajo de 15 años, y una licencia ambiental (ver adelante). Los otros 121 criterios son principalmente requisitos generales relativamente fáciles (Expoflores, 2016b), a excepción del criterio sobre calidad del agua efluente, que es estricto. Pero los cuatro criterios para una «gestión racional del agua» requieren solo una prueba de calidad si el agua se usa para consumo humano, una licencia para el uso de agua de riego bombeada y un registro del uso del agua de riego. Se puede obtener una certificación al cumplir el $80 \%$ de los criterios y así se pueden circunnavegar los criterios estrictos. La auditoría es realizada por Bureau Veritas Ecuador.

\section{Veriflora, SCS Global Services}

Veriflora es un programa desarrollado por SCS Global Services de EE.UU. con la visión de: «... proporcionar a los productores de flores cortadas ... de todo el mundo una hoja de ruta detallada para satisfacer el mercado emergente de productos sostenibles» (Jennifer Watters, SCS, comunicación personal, 20 de marzo de 2017). Las auditorías son realizadas por SCS Global Services. En 2012, 15 empresas de flores ecuatorianas tenían la certificación Veriflora (SCS 2012a). Su versión 3-1 (SCS 2012b), tiene nueve «elementos» con 152 criterios agrupados en dos «niveles». Las florícolas deben cumplir con todos los requisitos previos y los 50 «requisitos críticos». Además, se debe cumplir el $90 \%$ de los criterios $\mathrm{Ni}$ vel 1. A partir de los criterios Nivel 2 se debe cumplir el $80 \%$. Los obligatorios requieren el cumplimiento de la legislación local, como uso de pesticidas, mantenimiento de registros de recursos y uso y planificación de insumos. Los criterios están mayormente formulados de una manera no cuantitativa y multiinterpretable. Por ejemplo, el criterio 9.0 (Elemento 3), Nivel 1: dice:

«El Productor tiene que conservar agua a través de un uso eficiente de los sistemas de entrega de agua, métodos y tecnologías de conservación y seguimiento, y el establecimiento de prácticas de manejo de la calidad del agua ...».

Un criterio más estricto referente al riego es 9.1.2.6. (Nivel 2): «El productor instalará sistemas de riego que minimicen el consumo de agua mediante la aplicación directa a la zona de la raíz». Sin embargo, aunque todas las grandes florícolas cumplen al tener riego por goteo, esto no evita los efectos negativos de la extracción excesiva del recurso hídrico local o la competencia con los usuarios locales.

Los criterios para el uso del agua suelen referirse a nociones como «mayor eficiencia de uso", «conservación» y «ahorro». Esto parece sostenible; pero la floricultura requiere un cierto volumen de agua por unidad de producto. La reducción de la aplicación de este volumen de agua reducirá la producción y, por tanto, no se cambia la cantidad de agua utilizada por 
unidad de producto. Si antes se aplicaba más agua de la necesaria para el cultivo, esta agua se drenaba a las aguas superficiales o al acuífero, y estaba disponible para el uso de otros usuarios (Seckler, 1996), lo que implica un «ahorro» ficticio. Sin embargo, el discurso dominante de la «eficiencia» es poderoso y otorga legitimidad a la certificación y las empresas de flores certificadas, y simultáneamente quita poder a los pequeños floricultores que no usan tecnología avanzada. Esta es una forma de poder «oculto».

Los criterios Veriflora para las condiciones y derechos laborales son más estrictos que los criterios ambientales. En conclusión, aunque los criterios sobre el uso de plaguicidas prohíben que se usen los productos químicos más tóxicos (clase 1a y 1b), los criterios ambientales son permisivos y no parecen evitar los impactos negativos sobre el ambiente.

\section{Rainforest Alliance}

Desde 1992 Rainforest Alliance (RA) certifica la agricultura de plantaciones de banano y se ha ampliado a bosques, café y agricultura sostenible. En 2001, RA comenzó a certificar a los floricultores. El Sustainable Agricultural Standard de RA (RAS, Versión 1.2, julio de 2017) tiene cinco principios e incluye dos categorías: 1) 37 Criterios Críticos, y 2) 82 Criterios de Mejora Continua (Rainforest Alliance, 2018a). RAS tiene siete Criterios Críticos con respecto a la protección del ambiente: los criterios 3.1 a 3.7 se refieren a planes de control integrado de plagas, eliminación de aguas residuales y uso de aguas residuales. Se prohíbe usar organismos genéticamente modificados y aplicar ciertos agroquímicos desde aviones cuando los trabajadores están en el campo.

Para seguir certificadas las fincas demuestran mayor grado de cumplimiento a lo largo del tiempo de los Criterios de Mejora Continua. Se evalúa según el nivel de implementación de buenas prácticas, desde «Bueno» (Nivel C), con 65 \% después de un año, «Mejor» (Nivel B), con 50 \% después de tres años, y «Mejor» (Nivel A), 50 \% después de seis años. La Mejora Continua incluye el criterio relacionado con el agua 3.16: «Cualquier nuevo sistema de riego está diseñado para optimizar la productividad de cultivos o pastos mientras se minimiza el desperdicio de agua, erosión y salinización» (Nivel de rendimiento C), y el criterio 3.19: «... la granja demuestra reducciones en el agua utilizada para riego, procesamiento o producción de ganado por unidad de producto producido o procesado» (Nivel de rendimiento A). Estos criterios se relacionan con «ahorro» $y$ «eficiencia», y conducen a un ahorro de agua ficticio.

En 2018, un total de 45 grandes florícolas ecuatorianas tenían un certificado RA Sustainable Agricultural Standard (Rainforest Alliance, 2018b). La compañía de auditoría en todos los casos fue la compañía ecuatoriana CyD Certified.

\section{Fair trade}

La certificación de fair trade (comercio justo) comenzó en los 1980s por organizaciones sin fines de lucro europeas para ayudar a organizaciones de pequeños agricultores en el Sur 
Global. Las ventajas para estos eran, primero, tener contratos a largo plazo para vender en un mercado internacional y, segundo, un precio mínimo en caso de que el precio del mercado mundial cayera, pagos por adelantado, y un pago de prima adicional a la asociación de productores para usar en activos colectivos. En 1997 se inició la Fairtrade Labelling Organisation (FLO), denominada Fairtrade International (FI) desde 2009. FI certifica más de 300 productos en 66 países productores y vende en 120 países consumidores (Lyall, 2014). FLOCERT, ECOCERT y SCS Global Services son sus compañías de auditoría.

Desde 1994, ciertos productos provenientes de plantaciones pueden ser certificados con Fairtrade. Esta es una ruptura importante con la idea original. Aquí, los trabajadores deben tener ciertos derechos laborales garantizados, y la asociación de trabajadores de las plantaciones recibirá la prima Fairtrade. Primero el té y los bananos fueron certificados, y desde 2001 las flores. Tanto el té como las flores Fairtrade solo provienen de plantaciones. Además, se aplican criterios ambientales específicos. Las primeras flores ecuatorianas fueron certificadas con Fairtrade en 2002.

Hay resultados mixtos con la certificación Fairtrade en Ecuador. Raynolds (2014) encontró que, en general, los trabajadores de las florícolas ecuatorianas estaban satisfechos con las condiciones de trabajo y que las relaciones laborales eran más estables en las empresas certificadas. Pero también descubrió que a los trabajadores no se les permitía sindicarse. Grosse (2016) mostró que Fairtrade no prestaba suficiente atención en las florícolas ecuatorianas a las necesidades de las trabajadoras con niños:

«Las mujeres describieron ... buenas condiciones de trabajo, pero destacaron largas horas de trabajo, bajo salario y cuidado infantil inadecuado. Su trabajo requería que organizaran "estrategias económicas de género" privadas ... para asegurar un cuidado infantil que les permitiera brindar apoyo financiero a sus hijos, pero que limitaban su capacidad para cumplir con las expectativas de género sobre la atención infantil. A pesar de sus beneficios (...), el comercio justo proporciona un apoyo insuficiente para la atención en sus estándares y estructuras de producción. En este caso, no llega a facilitar arreglos de cuidado que permitan promover el desarrollo» (Grosse, 2016, 30).

Además, la mayoría de los consumidores de flores Fairtrade esperan ayudar a los pequeños productores: «Las ventas están siendo impulsadas porque el consumidor cree que los productos orgánicos son ... mejores para los pequeños productores» (Tamaki, 2013, 183). Pero, contrariamente a lo que la mayoría espera, las 12 florícolas certificadas Fairtrade en Ecuador son grandes, y las muchas pequeñas florícolas ecuatorianas no tienen y no pueden obtener una certificación porque no existe un estándar Fairtrade específico para ellos. Las pequeñas florícolas emplean mayormente a uno o dos trabajadores permanentes. Eso las descalifica como «pequeños productores» (véase www.fairtrade.net).

En Cayambe-Tabacundo, unas 170 pequeñas florícolas se organizaron en una asociación, AsoAgriPedro, que se describirá más adelante. De acuerdo con el espíritu de comercio justo, 
se esperaba que pudieran obtener un certificado. Sin embargo, esto no es posible: los pequeños productores de flores no pueden obtener el certificado Fairtrade como lo hacen los grandes, lo que quita poder a los primeros. Concomitantemente, esto muestra el poder oculto de la certificación: los consumidores de flores de comercio justo creen que les compran a asociaciones de pequeños productores, pero solo pueden comprar flores FairTrade de grandes empresas.

\section{Otros estándares}

El MPS holandés ofrece una variedad de estándares (MPS-ABC, MPS-SQ, MPS-GAP) con un grado variable de rigor. Hasta donde sabemos, solo un productor, la florícola holandesa Princess Roses, tenía las esquemas MPS-QS y MPS-GAP en 2018. Otro estándar es el Business Alliance for Secure Commerce (BASC) que garantiza, por ejemplo, contra el tráfico de drogas (BASC, 2018). Muchas grandes compañías tienen esta certificación, pero no incluye criterios estrictamente sociales o ambientales.

\section{Prácticas de Auditoría}

Las inspecciones son realizadas por empresas auditoras privadas. En Ecuador hay varias, nacionales e internacionales, que ofrecen estos servicios: CyD Certified, ECOCERT, FLOCERT, SCS Global Service, Bureau Veritas, Control Union, Ceres Certified y Kiwa BCS ÖKO-GARANTIE. Los costos de inspección son pagados por las florícolas. La auditoría de las empresas de flores generalmente tiene lugar una vez al año. Normalmente, las auditorías se anuncian de antemano. Durante la auditoría, los expertos visitan las instalaciones de producción. Si se obtienen resultados negativos de la auditoría, las empresas de flores se les da la oportunidad de mejorar sus prácticas. La compañía de auditoría generalmente permite entre 4 y 12 semanas para mostrar pruebas de dichos cambios para cumplir con la norma.

La competencia entre las empresas auditoras hace que estas se vuelvan más permisivas: los clientes tenderán a escoger a las que aplican criterios menos estrictos; una empresa que aplique rigurosamente las normas ambientales y sociales se enfrentará a un número decreciente de clientes de florícolas. Varios informes (Auld et al., 2008; Gulbrandsen, 2009; Klooster, 2010) sugieren que las auditorías privadas realizadas se enfrentan a muchos problemas, como son las múltiples interpretaciones de criterios y la susceptibilidad al fraude, lo que hace que la auditoría sea menos efectiva de lo que se afirma. La mayoría de las mejoras solicitadas se relacionan con las prácticas de documentación e informes, no con cambios requeridos en el terreno.

La certificación de flores es impulsada por la venta (el comprador): las florícolas (a diferencia de la mayoría de los productores orgánicos) solo están interesadas en cumplir con los estándares para obtener acceso al mercado. Como solo una relativamente pequeña parte del 
mercado requiere certificación, solo aquellos productores con prácticas relativamente buenas se molestan en obtener una certificación, mientras que las otras compañías venden en mercados menos exigentes. Esto implica que, para el sector de flores en Ecuador en general, la certificación no mejora las prácticas. Este efecto ha sido descrito para el manejo forestal por Auld et al. (2008) y Klooster (2010), y para el sector de la pesca por Gulbrandsen (2009). Esto también se observó mientras hacíamos la investigación de campo en Cayambe-Tabacundo: visitamos BellaRosa, la granja de flores en la que a todos les gustaría trabajar: dispositivos de aspiración de aire para matar insectos y todos los trabajadores con ropa protectora; pero esta era solo una de las pocas granjas dispuestas a ser entrevistadas. Otras grandes empresas fueron visitadas; sus invernaderos eran muy diferentes de los de BellaRosa: sustancias verdes y púrpuras goteaban y el aire se percibía estancado. Es difícil determinar si las prácticas de una florícola mejoraron debido al cumplimiento de las normas u otros factores como la motivación del propietario, la competencia por la fuerza de trabajo con los pequeños productores o las mejoras por la presión gubernamental, comunitaria o de las ONG.

Otra limitación de las inspecciones es que solo se examina la compañía, sin considerar su integralidad social y su entorno. Un empleado de la ONG Agronomes et Vétérinaires Sans Frontières (AVSF) en Ecuador comenta:

«Los esquemas de certificación a menudo solo miran cómo una empresa administra su agua. No estudian qué tipo de impacto tienen sus prácticas de gestión del agua en una escala mayor, en la comunidad o en la cuenca hidrográfica completa. Apenas miran más allá de sus paredes» (Sylvian Bleuze, comunicación personal, 21 de marzo de 2017).

Lo que los inspectores también dejan de lado es que parte de las flores es producida por pequeños productores no certificados y vendida a las florícolas certificadas.

Como las grandes florícolas no dependen de la certificación de terceros para vender flores, tienen bastante poder sobre las compañías auditoras. El sistema de auditoría de terceros por parte de compañías privadas competidoras se establece globalmente en un «espacio cerrado». Esta norma es una forma de "poder invisible» que empodera a las grandes florícolas.

\section{Regulación gubernamental Del SECTOR FLORícola}

El gobierno ecuatoriano tiene cuatro tipos de regulaciones para reducir los efectos negativos de la floricultura: licencias ambientales, ordenamiento territorial municipal, estándares ambientales y sociales nacionales y Agrocalidad.

\section{Licencia ambiental}

Se obtiene una licencia ambiental presentando una Evaluación de Impacto Ambiental (EIA) a la Autoridad Ambiental de Aplicación Responsable (AAAr), siendo esta generalmente el gobierno provincial y realizada por compañías especializadas. En el caso de Fio- 
rentina Flowers (MoyaGest, 2016), por ejemplo, el informe tiene 300 páginas, pero apenas proporciona información relevante sobre impactos ambientales o sociales; parece ser principalmente un «ejercicio de papel». Entonces, los estudios de EIA de las grandes florícolas son una forma de «poder oculto»: se presentan y aceptan como un instrumento para regular el sector, pero el estudio de los informes revela que los impactos y riesgos ambientales potenciales, como la contaminación del agua o la competencia con los pequeños propietarios por el agua, no se abordan.

\section{Ordenamiento territorial municipal}

El municipio otorga permisos para la construcción de invernaderos. Hay regulaciones y políticas de planificación del uso de la tierra locales. Un ejemplo de tal regulación es que los invernaderos de Pedro Moncayo no se deben construir a menos de $50 \mathrm{~m}$ de las casas. Sin embargo, las regulaciones difieren por municipio y el monitoreo es generalmente permisivo. En ambos municipios se construyen invernaderos muy cerca de las casas.

\section{Estándares nacionales}

El gobierno nacional tiene varios estándares que prohíben el uso de ciertos pesticidas, así como estándares de calidad del agua, condiciones de trabajo y salario mínimo. El aumento anual del salario mínimo en la última década es un avance significativo en la regulación gubernamental del sector. En 2007, cuando el presidente Correa llegó al poder, este era de 170 dólares al mes. Diez años más tarde ha aumentado a 375 dólares mensuales, uno de los más altos de Latinoamérica y que cubre la canasta básica. Como los salarios en otros países exportadores de flores son más bajos, la competencia se ha vuelto dura. Pero ha significado una situación socioeconómica mejorada para los trabajadores florícolas. En términos de regular el cumplimiento, la prohibición de pesticidas tóxicos parece efectiva (Breilh, 2007), los salarios pagados han seguido el ritmo del aumento del salario mínimo oficial y las condiciones de trabajo han mejorado (Soper, 2013).

\section{Agrocalidad}

Agrocalidad es un sistema de monitoreo de una agencia especializada. Los encuestados informan que Agrocalidad es la única agencia gubernamental que verifica in situ el cumplimiento monitoreando activamente la calidad de los productos de exportación. Principalmente controlan el número de áfidos y trips en las flores. Como todas las importaciones de flores a un país podrían bloquearse si se encuentran áfidos, a los exportadores de flores les interesa tener un control meticuloso. Agrocalidad inspecciona las grandes fincas semanalmente; cuando hay un problema con las flores, vienen dos veces por semana. Las granjas más pequeñas se controlan una vez al mes, pero hay más granjas pequeñas que las que Agrocali- 
dad tiene registradas y no todas se controlan. También vigilan el uso de productos químicos prohibidos. Un exinspector del FLP nos dijo que, aunque los agroquímicos más tóxicos están prohibidos, algunos trabajadores de las grandes florícolas utilizan las sustancias químicas «fuertes» para obtener rosas de mayor calidad exigidas por el mercado, incluso sin conocimiento de sus gerentes. La venta de agroquímicos no está bien controlada por el gobierno. Así, Agrocalidad establece la agenda solo monitoreando plagas y no problemas ambientales o sociales: esta es una forma de «poder oculto» ejercida por el gobierno nacional.

\section{LAS LUCHAS LOCALES POR LAS MEJORAS}

Desde la perspectiva de los programas de certificación, la reducción en el uso de pesticidas tóxicos y la mejora en las condiciones laborales son el resultado de la certificación. Desde la perspectiva del gobierno, este produce las mejoras al tener un control firme sobre las grandes compañías de flores. Pero estudiando los cambios en Cayambe-Tabacundo entendemos que las luchas locales para lograr mejoras también han sido cruciales. Tres se describen en esta sección: la lucha por el sistema de riego de Tabacundo; la lucha sobre los trabajadores (mano de obra) y las condiciones laborales entre los grandes y pequeños floricultores, el lanzamiento de AsoAgriPedro, y la organización de pequeños productores de flores.

\subsection{La lucha por el control del canal de riego}

El agua para las grandes florícolas en el área es proporcionada principalmente por la Acequia Tabacundo. Construida hace unos cien años, fue gestionada por la municipalidad de Pedro Moncayo hasta 2006. Las cerca de noventa grandes empresas con un total de 1.045 ha comparten el canal de riego con unos 2.500 pequeños propietarios, con un total de 2.400 ha. La asignación de agua ha sido muy desigual: anualmente las grandes florícolas reciben ocho millones de metros cúbicos, mientras que los muchos pequeños productores solo reciben dos millones de metros cúbicos (Hidalgo et al., 2017; Mena-Vásconez et al., 2017).

En 2006, los pequeños propietarios organizaron una protesta y tomaron el control del canal. Desde entonces, la asociación de pequeños propietarios de agua, CODEMIA, ha administrado el sistema de riego. Todavía asignan agua a las grandes florícolas porque muchos miembros de la familia de los pequeños agricultores trabajan en esas empresas, pero lentamente el agua se ha reasignado al sector de los pequeños agricultores. Las grandes florícolas han construido depósitos de agua de lluvia y perforado pozos. El mayor acceso al agua de riego - como resultado de la lucha de los pequeños propietarios por el control del sistema de riego- ayudó a muchos pequeños propietarios a poner en marcha su propio invernadero. Sin embargo, en dos de los cinco sectores de riego el consejo de usuarios prohíbe el establecimiento de invernaderos (Mena-Vásconez et al., 2017). Estas luchas locales son formas de 
«espacios reclamados» en la gobernanza del agua. La organización local de usuarios de agua aplicó un poder visible cuando marcharon para tomar el control del canal.

\subsection{Un mercado laboral competitivo}

Los pequeños productores de flores compiten con las grandes florícolas por los trabajadores. Esto obliga a las segundas a tratar a sus trabajadores mejor que antes: rociar menos insecticidas en presencia de los trabajadores y pagar salarios más altos. El ingeniero jefe de BellaRosa (53 ha) opina sobre las pequeñas empresas:

«La competencia con los pequeños es difícil. Es injusto porque hay muchas granjas ilegales pequeñas que no pagan regalías o no cumplen con los estándares de trabajo. Estas fincas deberían trabajar juntas para alcanzar los estándares y cumplir con todas las reglas. Si lo hicieran, no me estarías escuchando decir esto, porque entonces sería una competencia justa» (comunicación personal, 10 de marzo de 2017).

El avance de los pequeños productores de flores empodera a los trabajadores porque pueden reclamar un espacio para negociar las condiciones laborales. Muchos trabajadores prefieren comenzar su pequeño invernadero porque les flexibiliza el horario de trabajo y su espíritu empresarial puede verse recompensado. Otros prefieren el ingreso estable en una gran empresa, con un mercado laboral más ajustado que les posibilita una mejor negociación.

\subsection{El lanzamiento de AsoAgriPedro}

Desde principios de 2017, tras dos años de preparación, existe una organización de pequeños floricultores, AsoAgriPedro, con 170 propietarios que poseen en total 300 ha, aunque aparentemente solo alrededor de la mitad están activos en la asociación. Su administradora, Isabel Sánchez, ha descrito este proceso. Ella tenía una granja y vendía independientemente. Algunos vecinos preguntaron si podía ayudarlos a hacer esto y así comenzó la iniciativa con otras diez pequeñas florícolas. El municipio apoyó con tierras donde ubicaron sus oficinas e instalaciones de poscosecha. Desde abril de 2017, el gobierno de la provincia mostró su interés y se ofreció a ayudar a aumentar sus capacidades técnicas.

Cada mes se reúne la asamblea de miembros. Se discuten problemas y diseñan estrategias para el futuro. Comparten una instalación para poscosecha y gestionan la exportación de flores de manera conjunta. Hay una comisión de ventas que contacta con clientes y diseña estrategias comerciales. Además, hay una junta de 27 miembros, una unidad de administración de siete personas y 30 trabajadores de poscosecha. No hay requisitos para ser miembro de la asociación, solo estar registrados por Agrocalidad y tener los documentos legales. No hay tarifa de miembro, pero por flor vendida se pagan un par de centavos.

Muchos de los agricultores con los que se habló nunca habían oído de la asociación ni estaban interesados: «estás mejor solo que en una asociación con un compañero malo» (Raúl 
Moreno, comunicación personal, 10 de mayo de 2017). Otros, que tampoco habían oído hablar de ella, deseaban saber más porque veían ventajas. Marcos Gualavisí, propietario de un pequeño invernadero: «No hay ayuda, pero hay voluntad. Necesitamos ayuda» (comunicación personal, 8 de mayo de 2017). Sobre la pregunta sobre de dónde debe venir el cambio, Isabel Sánchez responde:

«El cambio debe venir desde abajo. Necesitamos darnos cuenta de que no somos pequeños productores. Pensar así es un gran error. Como asociación, somos el mayor negocio de flores de Ecuador, lo que hace que sea aún más importante para nosotros que nos centremos en el aspecto social de las cosas y nos preocupemos, por ejemplo, de los productos químicos que utilizamos».

Isabel Sánchez admitió que las condiciones de trabajo podrían no ser tal como lo exige la ley, ya que, por ejemplo, los niños ayudan en los invernaderos. La certificación podría ser difícil, pero la asociación no busca eso: «Las grandes compañías necesitan mantener su glamur, por eso las certificaciones como Fairtrade pueden atraerlas, pero para nosotros eso no es importante hoy».

Ana Farinango con su granja, «donde el trabajo es 24/7»: «No tengo tiempo para relacionarme con otras pequeñas empresas» (comunicación personal, 4 de marzo de 2017). Para ella, sus flores son «como sus hijas» y, por lo tanto, también tiene miedo de "compartir» sus flores. Sin embargo, dijo que estaría interesada en una asociación. Un año después, Ana Farinango (comunicación personal, 10 de marzo de 2018) ha superado sus temores de asociarse y lidera un grupo de seis floricultoras en su comunidad. Su objetivo es establecer una asociación formal, pero por el momento está contenta con que todos contribuyan a construir sus propias instalaciones de poscosecha; está invirtiendo el pequeño excedente que logró después de una temporada de San Valentín exitosa, ya que no puede obtener más crédito del banco nacional. Allí tendrá instalaciones de almacenamiento en frío para recolectar y procesar rosas propias y del grupo y más allá. Además de desarrollarse, quiere que sus vecinos eviten los altos riesgos y la molestia de depender de los comerciantes de flores que ella ha sufrido durante años. Este asociacionismo podría ser una tendencia, pero es temprano para decirlo.

AsoAgriPedro y otras asociaciones similares podrían entenderse como espacio reclamado que abre el ángulo de maniobra y ganancias financieras para los pequeños propietarios, aunque no tienen poder al no obtener el certificado de comercio justo. Si pueden exportar directamente, sin grandes florícolas o comerciantes, los pequeños agricultores ganan económicamente. Sin embargo, el éxito precario de los pequeños también podría analizarse como una contribución al poder invisible (o gubernamentalidad) de los discursos económicos que valoran el dinero sobre la soberanía alimentaria y el emprendimiento privado sobre la identidad como productores colectivos de alimentos (para este análisis, véase Mena et al., 2016). Este discurso empodera a los grandes y pequeños empresarios de flores sobre los pequeños productores de alimentos que necesitan acceso al agua de riego para sus cultivos alimentarios. 


\subsection{Luchas por el agua, las normas y el poder de decisión}

Mena et al. (2016, 2017) y Hidalgo et al. (2017) muestran cómo el afán de modernizar la estructura agraria a través de grandes empresas comerciales de exportación de flores y el desarrollo de cadenas de agronegocios transnacionales es parte del «plan de salvación» utópico neoliberal. La producción de rosas concentra la mayor parte del agua de riego disponible, en términos de cantidad y seguridad de servicio, y es utilizada por los agronegocios y pequeños productores de rosas en detrimento de los cultivos tradicionales de ciclo corto (Boelens y Vos, 2012; Vos y Boelens, 2018). Las empresas exportadoras pueden ofrecer pagos más altos por el acceso al agua y el mantenimiento de la infraestructura, un incentivo perverso que ha provocado prácticas de asignación de agua inequitativas. El creciente uso del agua para la agricultura de exportación de flores en el área de Cayambe-Tabacundo ha afectado profundamente los ecosistemas locales y el tejido sociocultural de las comunidades indígenas. Como consecuencia, conduce a la transformación de las sociedades indígenas no solo en términos de transformación de sus modos de acceso y gestión del agua, sino también en relación con el cambio de sus realidades subjetivas a las de los productores racionales «modernos» e individualizados.

Fundamentalmente, como Hidalgo et al. (2017) describen, la seguridad hídrica local está nuevamente y cada vez más amenazada por la captura de agua, ahora con flujos de agua «virtuales», pero muy reales y materiales, en un contexto dominado por las empresas de agroexportación. Controlan el agua mediante fuerzas materiales y tecnológicas que alteran los flujos de agua, el poder económico, la influencia en las políticas nacionales y las prerrogativas para establecer normas de producción aplicadas a nivel internacional, así como la necesidad de que la población local venda su fuerza de trabajo. Por lo tanto, el «agua» se ha convertido en un tema prominente en las protestas sociales que responden en contra de la marginación económica, la discriminación étnica y la gobernanza no democrática. Para contrarrestar la usurpación de sus medios de subsistencia, territorios y fuentes de agua las comunidades indígenas participan en luchas legislativas y, ocasionalmente, en conflictos abiertos. La aplicación continua y la proliferación de «marcos de derechos de agua indígenas» en el control diario del agua es una forma poderosa de impugnar las estructuras de poder dominantes y fomentar sus reclamos sobre los recursos hídricos y la legitimidad. Estas luchas cuestionan la invasión de los derechos de agua colectivos de los usuarios indígenas y, por lo tanto, van más allá de las «luchas por recursos». Los pueblos indígenas exigen el reconocimiento de los derechos territoriales, la distribución equitativa del agua y la legitimación de las autoridades y los marcos normativos locales, crean «espacios reclamados» (Hoogesteger, 2012; Mena et al., 2016; Hidalgo et al., 2017). 


\section{Discusión y CONCLUSIÓN}

Existen muchos factores para explicar los cambios en los impactos sociales y ambientales de la producción de flores de exportación. Las políticas de RSE adoptadas por las empresas privadas a menudo incluyen estándares ambientales y sociales que deben garantizar cierto límite a los impactos sociales y de salud para los trabajadores y un impacto ambiental mínimo para los lugares de producción y su entorno. Pero la contribución real de esos estándares a las mejoras es difícil de establecer. Muchas otras dinámicas influyen en los impactos socioambientales de la agricultura de exportación.

El estudio de caso de la floricultura de exportación a gran escala en el norte de Ecuador muestra que más allá de la certificación privada varios factores han contribuido a la mejora de las prácticas de la floricultura ecuatoriana y su gobernanza del agua: primero, el aumento oficial del salario mínimo; segundo, la interacción y competencia entre grandes y pequeños productores para ofrecer mejores condiciones socioeconómicas y ambientales y así atraer colaboradores en sus fincas; tercero, las regulaciones ambientales de los gobiernos nacionales, provinciales y municipales; cuarto; la presión de las ONG locales; y quinto, de manera fundamental, las luchas de las comunidades campesinas y las asociaciones de usuarios por la reasignación del agua de riego y el respeto a su dignidad.

Los estándares privados como los analizados han tenido relativamente poco efecto en las prácticas florícolas. La demanda de flores certificadas se limita al norte de Europa y a ciertos compradores en EE.UU., mientras que una gran proporción de las flores se exporta a otras regiones que no exigen certificación. Como un efecto negativo, los estándares otorgan poder a las empresas certificadas a medida que obtienen acceso al mercado y un uso legítimo del agua por parte de los productores de flores a gran escala, sin tener en cuenta el entorno más allá de los límites de la finca. El discurso de regulación privada detrás de los estándares es una forma de poder invisible: una gubernamentalidad neoliberal (Boelens et al., 2014, 2015; Vos y Boelens, 2014, 2018). Los estándares y las reglamentaciones de EIA del gobierno representan espacios cerrados dentro de nuestro análisis del cubo de poder, donde las normas se establecen sin la participación de las partes interesadas afectadas. Las normas internalizadas (como la de «la eficiencia es buena») actúan como formas invisibles de poder, empoderando a las grandes compañías de flores que tienen licencias y certificados.

Hemos mostrado la contradicción con respecto al discurso generalizado sobre certificación $=$ comercio justo $=$ justicia socioambiental: en Ecuador, relativamente pocas grandes empresas están certificadas y al contrario de las expectativas de los consumidores, los pequeños productores no pueden tener esos certificados. Así, esta certificación otorga un poder oculto para las grandes compañías y comerciantes certificados. Desde abril de 2017, se ha establecido una pequeña asociación de productores de flores. Si bien operan con el espíritu de comercio justo, los estándares oficiales de «comercio justo» no les permiten certificarse. 
Para sus luchas por el agua, es fundamental que los pequeños productores y las comunidades campesinas del Cayambe-Tabacundo no sólo se organicen al nivel local, reclamando espacios locales de decisión. Para reclamar el agua, un ambiente sano, tener derecho y voz en la gobernanza y ver que sus normas y formas organizativas estén aceptadas y tomadas en cuenta, existe la necesidad de organizarse mediante vínculos locales-nacionales-globales, ya que los procesos de acumulación de poder de decisión y la desposesión de agua también forman parte de procesos más amplios y, como en el caso de las flores, hasta transnacionales.

\section{Agradecimientos}

Agradecemos a Kati Mayfield, de la Universidad de Aalto, Finlandia por la revisión de una versión temprana del manuscrito.

\section{REFERENCIAS}

Auld, G., Gulbrandsen, L.H. y McDermott, C.L. (2008): Certification schemes and the impacts on forests and forestry. Annual review of environment and resources 33, 187-211.

Ayala Mora, E. (ed.) (2008): Manual de Historia del Ecuador. Tomo 1. Corporación Editora Nacional, Quito.

BASC (2018): Business Alliance for Secure Commerce, http://www.wbasco.org/en.

Baumgarten, B. y Ullrich, P. (2012): Discourse, Power, and Governmentality. Social Movement Research with and beyond Foucault. En Roose, J. and Dietz, H. (eds.): Social Theory and Social Movements. Springer, Wiesbaden, pp. 13-38.

Bloemisterij (2017): Ecuador heeft nog een lange weg te gaan in duurzaamheid (Ecuador has a long way to go to sustainability), 6 Nov 2017, https://www.hortipoint.nl/vakbladvoordebloemisterij/ecuadorheeft-nog-een-lange-weg-te-gaan-in-duurzaamheid/.

Boelens, R. y J. Vos (2012) The danger of naturalizing water policy concepts. Water productivity and efficiency discourses from field irrigation to virtual water trade. Journal of Agricultural Water Management, 108, 16-26, doi:10.1016/j.agwat.2011.06.013.

Boelens, R., J. Hoogesteger y J. C. Rodriguez de Francisco (2014): Commoditizing Water Territories: The clash between Andean water rights cultures and Payment for Environmental Services policies. Capitalism Nature Socialism, 25(3), 84-102, doi:10.1080/10455752.2013.876867.

Boelens, R., J. Hoogesteger y M. Baud (2015): Water reform governmentality in Ecuador: neoliberalism, centralization and the restraining of polycentric authority and community rule-making, Geoforum, 64, 281-291, doi: 10.1016/j.geoforum.2013.07.005.

BREILH, J. (2007): Nuevo modelo de acumulación y agroindustria: las implicaciones ecológicas y epidemiológicas de la floricultura en Ecuador. Ciênc. Saúde Coletiva, 12(1), 91-104. 
Breilh, J., Campaña, M., Felicita, O., Hidalgo, F., Lourdes Larrea, M., Sánchez, D., Straka, N., y Yassi A. (2009): Consolidación del estudio sobre la relación entre impactos ambientales de la floricultura, patrones de exposición y consecuencias en comunidades de la cuenca del Granobles (Sierra Norte, Ecuador). Informe técnico final, Centro de Estudios y Asesoría en Salud - CEAS, Quito.

Expoflores (2016a): Certificación FlorEcuador, Reglamento General para empresas de producción, exportación y comercialización de flores, Versión 2.2, Junio 2016, 29p, http://expoflores.com/ wp-content/uploads/2016/12/reglamento_general_FlorEcuador.pdf.

Expoflores (2016b): Certificación FlorEcuador, Lista de Chequeo 21p. http://expoflores.com/wp-content/uploads/2016/12/lista_chequeo_FlorEcuador_Certified_julio16.pdf.

Foucault, M. (1991): Governmentality. En I Burchell, G., Gordon, C. y Miller, P: The Foucault effect: studies in governmentality: with two lectures by and an interview with Michel Foucault. The Foucault Effect: Studies in Governmentality. The University of Chicago Press, Chicago, pp. 87104.

Foucault, M. (1995 (1975)): Discipline and punish. The birth of the prison, Vintage Books, New York.

Franze, J. y Ciroth, A. (2011): A comparison of cut roses from Ecuador and the Netherlands' International Journal Life Cycle Asses, 16, 366-379.

Gaventa, J. (2006): Finding the spaces for change: a power analysis, IDS bulletin, 37(6), 23-33.

GADIP (Gobierno Autónomo Descentralizado Intercultural y Plurinacional del Municipio de Cayambe) (2015): Somos Cayambe, Cayambe.

Grosse, C.E. (2016): Fair care? How Ecuadorian women negotiate childcare in fair trade flower production, Women's Studies International Forum, 57, 30-37.

Gulbrandsen, L.H. (2009): The emergence and effectiveness of the Marine Stewardship Council, Marine Policy, 33(4), 654-660.

Hidalgo, J. P., Boelens, R. y Vos, J. (2017): De-colonizing water. Dispossession, water insecurity, and Indigenous claims for resources, authority, and territory. Water History, 9(1), 67-85.

Hoogesteger, J. (2012). Trans-Forming Social Capital Around Water: Water User Organizations, Water Rights, and Nongovernmental Organizations in Cangahua, the Ecuadorian Andes. Society and Natural Resources, 26, 60-74.

Hughes, A. (2001): Global Commodity Networks, Ethical Trade and Governmentality: Organizing Business Responsibility in the Kenyan Cut Flower Industry. Transactions of the Institute of British Geographers, 26(4), 390-406.

Instituto de Ecología y Desarollo de las Comunidades Andinas, IEDECA (1999): Impacto de la floricultura en los campesinos de Cayambe. IEDECA, Quito.

KLooster, D. (2010): Standardizing sustainable development? The Forest Stewardship Council's plantation policy review process as neoliberal environmental governance. Geoforum, 41(1), 117-129.

Korovkin, T. (2003): Cut-Flower Exports, Female Labor, and Community Participation in Highland Ecuador, Latin American Perspectives, 30(4), 18-42.

Langen, N. y Adenaeuer, L. (2013): Where does the fair trade price premium go? Confronting consumers' request with reality. Social Enterprise Journal, 9(3), 293-314. 
Lemke, T. (2007): An indigestible meal? Foucault, governmentality and state theory, Distinktion: Journal of Social Theory, 8(2), 43-64.

Lyall, A. (2014): Assessing the impacts of fairtrade on worker-defined forms of empowerment on Ecuadorian flower plantations, Final report commissioned by Fairtrade International and Max Havelaar-Foundation.

LyNCH B.D. (2012): Vulnerabilities, competition and rights in a context of climate change toward equitable water governance in Peru's Rio Santa Valley, Global Environmental Change, 22(2), 364373.

Martínez, L. (2014): De la hacienda al agronegocio: agricultura y capitalismo en Ecuador. En: G. Almeyra, L.C. Bórquez, J. M. Mendes Pereira y C. W. Porto-Gonçalves (coord.) (2014). Capitalismo: tierra y poder en América Latina (1982-2012) Vol II. México: Universidad Autónoma Metropolitana, pp. 123-158.

Mena-VÁsconez, P., Boelens, R. y Vos, J. (2016): Food or flowers? Contested transformations of community food security and water use priorities under new legal and market regimes in Ecuador's highlands. Journal of Rural Studies, 44, 226-238.

Mena-Vásconez, P., Vincent, L., Vos, J. y Boelens, R. (2017): Fighting over water values: diverse framings of flower and food production with communal irrigation in the Ecuadorian Andes. Water International, 42(4), 443-461.

MoyaGest (2016): Estudio de impacto ambiental ex post y plan de manejo ambiental finca florícola «Fiorentina Flowers S.A.», 300 pp., http://fiorentinaflowers.com/wp-content/uploads/2017/05/Fiorentina-Flowers-Environmental-Plan-EsIA-Ex-Post-FIORENTINA-2016-002.pdf.

Mutersbaugh, T., Klooster, D., Renard, M.C. y Taylor, P. (2005): Certifying rural spaces: quality-certified products and rural governance. Journal of Rural Studies, 21(4), 381-388.

Rainforest Alliance (2018a): Cultivating Earth-Friendly Flowers, webpage: https://www.rainforest-alliance.org/articles/rainforest-alliance-certified-ferns-flowers.

Rainforest Alliance (2018b): Rainforest Alliance Sustainable Agriculture Standard, 2017, Version 2.1, https://www.rainforest-alliance.org/business/sas/wp-content/uploads/2017/11/03_rainforest-alliance-sustainable-agriculture-standard_en.pdf.

Raynolds, L.T. (2014): Fairtrade, certification, and labor: global and local tensions in improving conditions for agricultural workers, Agriculture and Human Values, 31(3), 499-511.

Roa-García, M.C. (2014): Equity, efficiency and sustainability in water allocation in the Andes: Trade-offs in a full world, Water Alternatives 7(2), 298-319.

Rodríguez-de-Francisco, J. C. y Boelens, R. (2016): PES hydrosocial territories: de-territorialization and re-patterning of water control arenas in the Andean highlands. Water International, 41(1), 140-156, https://doi.org/10.1080/02508060.2016.1129686.

SAwers, L. (2005): Sustainable Floriculture in Ecuador. International Journal of Economic, Social, and Environmental Sustainability, 1, 3-9.

SCS Global Services (2012a): Veriflora certified producers, handlers and approved input materials manufacturers, $8 \mathrm{pp}$. 
SCS Global Services (2012b): Veriflora. Certification of sustainably grown cut flowers and potted plants. requirements for growers and handlers, Version 3-1. 148p. https:/www.scsglobalservices. com/files/standards/SCS_STN_Veriflora_V3-1_100912.pdf.

SECKLER, D. (1996): The new era of water resources management: From «dry» to «wet» water savings. Colombo, Sri Lanka: International Irrigation Management Institute (IIMI); IWMI. iii, 17p. (IIMI Research Report 1).

SIPAE (2016) Impactos de la certificación FLP (Flower Label Program) en el sector florícola ecuatoriano, Quito.

Soper, R. (2013) Reclaiming Development: Indigenous Community Organizations and the Flower Export Industry in the Ecuadorian Highlands. En M. BAcker (Ed.): Indigenous and Afro-Ecuadorians Facing the Twenty-First Century. Cambridge Scholars Publishing, Newcastle upon Tyne, pp. 128-149.

TAмaki, R. (2013): Consumer's perception of fair trade coffee in Australia and Japan (Doctoral dissertation). Curtin University.

Tenenbaum, D. (2002): Would a rose not smell as sweet?, Environmental Health Perspectives, 110(5), A240-A247.

USDA Foreign Agricultural Service (2009): Ecuador Fresh Flower Industry Situation (EC9006). Office of Global Analysis, Quito.

Vos, J. y Boelens, R. (2014): Sustainability standards and the water question. Development and Change, 45(2), 205-230.

Vos, J. y Boelens, R. (2018):. Neoliberal water governmentalities, virtual water trade, and contestations. In R. Boelens, T. Perreault, J. Vos (Eds.): Water Justice, pp. 283-301. Cambridge University Press, Cambridge.

Cómo citar este artículo:

Mena-Vásconez, P., Vos, J., van Ommen, P. y Boelens, R.(2018). Flores, acaparamiento del agua y responsabilidad empresarial social: certificación de la producción de rosas y reclamos por la justicia ambiental en el Ecuador. Cuadernos de Geografía, 101, 189-214. https://doi.org/10.7203/CGUV.101.13727

\section{(c) (1) $\Theta \Theta$}

Este obra está bajo una licencia de Creative Commons Reconocimiento-NoComercial-SinObraDerivada 4.0 Internacional. 